\title{
Critical Blow-Up and Global Existence for Discrete Nonlinear $p$-Laplacian Parabolic Equations
}

\author{
Soon-Yeong Chung \\ Department of Mathematics and Program of Integrated Biotechnology, Sogang University, Seoul 121-742, Republic of Korea \\ Correspondence should be addressed to Soon-Yeong Chung; sychung@sogang.ac.kr
}

Received 15 October 2014; Accepted 24 November 2014; Published 14 December 2014

Academic Editor: Hassan A. El-Morshedy

Copyright (C) 2014 Soon-Yeong Chung. This is an open access article distributed under the Creative Commons Attribution License, which permits unrestricted use, distribution, and reproduction in any medium, provided the original work is properly cited.

The goal of this paper is to investigate the blow-up and the global existence of the solutions to the discrete $p$-Laplacian parabolic equation $u_{t}(x, t)=\Delta_{p, w} u(x, t)+\lambda|u(x, t)|^{p-2} u(x, t),(x, t) \in S \times(0, \infty), u(x, t)=0,(x, t) \in \partial S \times(0, \infty), u(x, 0)=u_{0}$, depending on the parameters $p>1$ and $\lambda>0$. Besides, we provide several types of the comparison principles to this equation, which play a key role in the proof of the main theorems. In addition, we finally give some numerical examples which exploit the main results.

\section{Introduction}

A discrete $p$-Laplacian parabolic equation (a reactiondiffusion system) has found many applications in chemical reactions and biological phenomena. A typical example is an autocatalytic chemical reaction between several chemicals in which the concentration of each chemical grows (or decays) due to diffusion and difference of concentration. In general, many of such phenomena are modeled by

$$
u_{t}(x, t)=\Delta_{p, w} u(x, t)+\lambda|u(x, t)|^{q-1} u(x, t), \quad x \in S,
$$

with some boundary and initial conditions where $S$ is the set of chemicals and $p>1, q>0$. Here, $\Delta_{p, w}$ is the discrete $p$-Laplace operator on a network $S$, defined by

$$
\Delta_{p, w} f(x):=\sum_{y \in \bar{S}}|f(y)-f(x)|^{p-2}[f(y)-f(x)] \omega(x, y) .
$$

For the continuous case, solutions to the initial value problem of (1) may blow up in a finite time or exist globally for all time, depending on the parameters $p>1, q>0$, and $\lambda \in \mathbb{R}$ (see [1-7] and see the book [2] for more information about the various blow-up phenomena). In particular, the case where $p=q-1>1$ has been known as the critical case at which solutions may blow up or exist globally, depending on the parameter $\lambda$.
The goal of this paper is to investigate the blow-up and the global existence of the solutions to the initial value problem of the discrete $p$-Laplacian parabolic equation

$$
u_{t}(x, t)=\Delta_{p, w} u(x, t)+\lambda|u(x, t)|^{p-2} u(x, t), \quad x \in S,
$$

on a network $S$ with boundary, which is the critical case of (1).

The continuous case of (3) has been studied by many authors. For example, the case where $1<p<2$ was studied in $[3,6]$ and the case where $p>2$ was studied in $[3,4]$.

For the discrete case of (1), the authors studied the general theory of the equation for the case $p=2$ in [8] and Xin et al. studied the blow-up solutions and global solutions for the case where $p=2$ and $\lambda=1$ in [9]. On the other hand, for the case $\lambda \leq 0$ in (1), Chung et al. [10,11] investigate the positive solutions and extinctive solutions, depending on the parameters $p$ and $q$. But, the case where $p>1$ and $\lambda>0$ in (3) has not been studied yet. Thus, the purpose of this paper is to give a complete solution to such a problem about (3).

The main results of this paper are summarized as follows.

Theorem 1. (i) For $p>2$ and $\lambda>\lambda_{0}$, the solution to

$$
\begin{array}{r}
u_{t}(x, t)=\Delta_{p, w} u(x, t)+\lambda|u(x, t)|^{p-2} u(x, t), \\
(x, t) \in S \times(0, \infty),
\end{array}
$$




$$
\begin{aligned}
& u(x, t)=0, \quad(x, t) \in \partial S \times(0, \infty), \\
& u(x, 0)=u_{0}
\end{aligned}
$$

blows up in a finite time $T$, for every nonnegative and nontrivial initial data $u_{0}$.

Here, $\lambda_{0}$ is the first eigenvalue of the p-Laplace operator $\Delta_{p, w}$ on $S$. Moreover, it is shown that the blow-up rate of the solution is estimated by

$$
\begin{array}{r}
\max _{x \in S} u(x, t) \geq\left[(p-2)\left(\lambda-\lambda_{0}\right)(T-t)\right]^{-(1 /(p-2))}, \\
t>0 .
\end{array}
$$

(ii) For $p>2$ and $\lambda \leq \lambda_{0}$, the solution to (4) is global, for every nonnegative initial data $u_{0}$.

(iii) For $1<p \leq 2$, the nonnegative solution to (4) is global for every nonnegative initial data $u_{0}$. In particular, when $1<$ $p<2$ and $\lambda<\lambda_{0}$, there exists $T>0$ (extinction time) such that $u(x, t) \equiv 0$ for all $t \geq T$.

Besides, we provide several types of the comparison principles to (4) in Section 2, which play a key role in the proof of the main theorems and we give some numerical examples in the final section which exploit the main results.

\section{Preliminaries and Discrete Comparison Principles}

In this section, we start with some definitions of graph theoretic notions frequently used throughout this paper (see $[8,12,13]$ for more details).

For a graph $G=G(V, E)$ we mean finite sets $V$ of vertices (or nodes) with a set $E$ of two-element subsets of $V$ (whose elements are called edges). The set of vertices and edges of a graph $G$ is sometimes denoted by $V(G)$ and $E(G)$, or simply $V$ and $E$, respectively. Conventionally, we denote by $x \in V$ or $x \in G$ the facts that $x$ is a vertex in $G$.

A graph $G$ is said to be simple if it has neither multiple edges nor loops, and $G$ is said to be connected if, for every pair of vertices $x$ and $y$, there exists a sequence (called a path) of vertices $x=x_{0}, x_{1}, \ldots, x_{n-1}, x_{n}=y$ such that $x_{j-1}$ and $x_{j}$ are connected by an edge (called adjacent) for $j=1, \ldots, n$.

A graph $S=S\left(V^{\prime}, E^{\prime}\right)$ is said to be a subgraph of $G(V, E)$ if $V^{\prime} \subset V$ and $E^{\prime} \subset E$.

A weight on a graph $G$ is a function $\omega: V \times V \rightarrow[0,+\infty)$ satisfying

(i) $\omega(x, x)=0, x \in V$,

(ii) $\omega(x, y)=\omega(y, x)$ if $x \sim y$,

(iii) $\omega(x, y)=0$ if and only if $x+y$.

Here, $x \sim y$ means that two vertices $x$ and $y$ are connected (adjacent) by an edge in $E$. A graph associated with a weight is said to be a weight graph or a network.

For a subgraph $S$ of a graph $G(V, E)$, the (vertex) boundary $\partial S$ of $S$ is the set of all vertices $z \in V \backslash S$ but adjacent to some vertex in $S$; that is,

$$
\partial S:=\{z \in V \backslash S \mid z \sim y \text { for some } y \in S\} .
$$

By $\bar{S}$ we denote a graph, whose vertices and edges are in both $S$ and $\partial S$. Throughout this paper, all subgraphs $S$ and $\bar{S}$ in our concern are assumed to be simple and connected.

For a function $u: \bar{S} \rightarrow \mathbb{R}$, the discrete p-Laplacian $\Delta_{p, \omega}$ on $S$ is defined by

$$
\Delta_{p, \omega} u(x):=\sum_{y \in \bar{S}}|u(y)-u(x)|^{p-2}[u(y)-u(x)] \omega(x, y)
$$

for $x \in S$.

The following lemmas are useful throughout this paper.

Lemma 2 (see $[14,15]$ ). For functions $f, g: \bar{S} \rightarrow \mathbb{R}$, one has the following:

$$
\begin{aligned}
& \text { (i) } 2 \sum_{x \in \bar{S}} g(x)\left[-\Delta_{p, \omega} f(x)\right]=\sum_{x, y \in \bar{S}}|f(y)-f(x)|^{p-2} \\
& {[f(y)-f(x)] \cdot[g(y)-g(x)] \omega(x, y) \text {; }} \\
& \text { (ii) } 2 \sum_{x \in \bar{S}} f(x)\left[-\Delta_{p, \omega} f(x)\right]=\sum_{x, y \in \bar{S}}|f(x)-f(y)|^{p} \omega(x, \\
& \quad y) .
\end{aligned}
$$

Lemma 3 (see $[14,15]$ ). For $p>1$, there exist $\lambda_{0}>0$ and $\phi_{0}(x)>0, x \in S$, such that

$$
\begin{aligned}
-\Delta_{p, \omega} \phi_{0}(x) & =\lambda_{0} \phi_{0}^{p-1}(x), \quad x \in S, \\
\phi_{0}(x) & =0, \quad x \in \partial S, \\
\sum_{x \in S} \phi_{0}^{p}(x) & =1 .
\end{aligned}
$$

Moreover,

$$
\begin{aligned}
\lambda_{0} & =\min _{u \in \mathscr{A}, u \neq 0} \frac{(1 / 2) \sum_{x, y \in \bar{S}}|u(x)-u(y)|^{p} \omega(x, y)}{\sum_{x \in \bar{S}}|u(x)|^{p}} \\
& \leq \sum_{y \in \bar{S}} \omega(x, y), \quad x \in S,
\end{aligned}
$$

where $\mathscr{A}:=\{u: \bar{S} \rightarrow \mathbb{R} \mid u=0$ on $\partial S\}$.

In the above, the number $\lambda_{0}$ is called the first eigenvalue of $\Delta_{p, \omega}$ on a network $\bar{S}$ with corresponding eigenfunction $\phi_{0}$ (see $[12,13]$ for the spectral theory of the Laplacian operators).

The rest of this section is devoted to proving the comparison principles for the discrete $p$-Laplacian parabolic equation

$$
\begin{aligned}
& u_{t}(x, t)=\Delta_{p, \omega} u(x, t)+\lambda|u(x, t)|^{p-2} u(x, t), \\
& (x, t) \in S \times(0, \infty), \\
& u(x, t)=0, \quad(x, t) \in \partial S \times(0, \infty), \\
& u(x, 0)=u_{0}, \quad x \in \bar{S},
\end{aligned}
$$

where $\lambda>0$ and $p>1$, in order to study the blow-up occurrence and global existence, which we begin in the next section.

Now, we state the comparison principles and some related corollaries. 
Theorem 4. Let $T>0$ (T may be $+\infty), \lambda>0$, and $p \geq 2$. Suppose that real-valued functions $u(x, \cdot), v(x, \cdot) \in C[0, T)$ are differentiable in $(0, T)$ for each $x \in \bar{S}$ and satisfy

$$
\begin{array}{r}
u_{t}(x, t)-\Delta_{p, \omega} u(x, t)-\lambda|u(x, t)|^{p-2} u(x, t) \\
\geq v_{t}(x, t)-\Delta_{p, \omega} v(x, t)-\lambda|v(x, t)|^{p-2} v(x, t), \\
(x, t) \in S \times(0, T),
\end{array}
$$

$$
\begin{aligned}
& u(x, t) \geq v(x, t), \quad(x, t) \in \partial S \times[0, T), \\
& u(x, 0) \geq v(x, 0), \quad x \in \bar{S} .
\end{aligned}
$$

Then $u(x, t) \geq v(x, t)$ for all $(x, t) \in \bar{S} \times[0, T)$.

Proof. Let $T^{\prime}>0$ be arbitrarily given with $T^{\prime}<T$. Then by the mean value theorem, for each $x \in S$ and $0 \leq t \leq T^{\prime}$,

$$
\begin{aligned}
& |u(x, t)|^{p-2} u(x, t)-|v(x, t)|^{p-2} v(x, t) \\
& \quad=(p-1)|\xi(x, t)|^{p-2}[u(x, t)-v(x, t)]
\end{aligned}
$$

for some $\xi(x, t)$ lying between $u(x, t)$ and $v(x, t)$. Then it follows from (11) that we have

$$
\begin{aligned}
u_{t} & -\Delta_{p, \omega} u-\lambda(p-1)|\xi(x, t)|^{p-2} u(x, t) \\
& \geq v_{t}-\Delta_{p, \omega} v-\lambda(p-1)|\xi(x, t)|^{p-2} v(x, t)
\end{aligned}
$$

for all $(x, t) \in S \times\left(0, T^{\prime}\right]$. Let $\widetilde{u}, \widetilde{v}: \bar{S} \times\left[0, T^{\prime}\right] \rightarrow \mathbb{R}$ be the functions defined by

$$
\begin{aligned}
& \widetilde{u}(x, t):=e^{-2 \lambda(p-1) L t} u(x, t), \\
& \widetilde{v}(x, t):=e^{-2 \lambda(p-1) L t} v(x, t),
\end{aligned}
$$

where $L:=\max _{|r| \leq M}\left|r^{p-2}\right|$ and $M:=\max _{x \in \bar{S}, t \in\left[0, T^{\prime}\right]}\{|u(x, t)|$, $|v(x, t)|\}$.

Then inequality (13) can be written as

$$
\begin{aligned}
& \tilde{u}_{t}(x, t)-\widetilde{v}_{t}(x, t)-e^{2 \lambda L(p-1)(p-2) t} \\
& \quad \times\left[\Delta_{p, \omega} \widetilde{u}(x, t)-\Delta_{p, \omega} \widetilde{v}(x, t)\right]+\lambda(p-1) \\
& \quad \times\left[2 L-|\xi(x, t)|^{p-2}\right][\widetilde{u}(x, t)-\widetilde{v}(x, t)] \geq 0
\end{aligned}
$$

for all $(x, t) \in S \times\left(0, T^{\prime}\right]$. Since $\bar{S} \times\left[0, T^{\prime}\right]$ is compact, there exists $\left(x_{0}, t_{0}\right) \in \bar{S} \times\left[0, T^{\prime}\right]$ such that

$$
(\widetilde{u}-\widetilde{v})\left(x_{0}, t_{0}\right)=\min _{x \in \bar{S}} \min _{0 \leq t \leq T^{\prime}}(\tilde{u}-\widetilde{v})(x, t) .
$$

Then we have only to show that $(\widetilde{u}-\widetilde{v})\left(x_{0}, t_{0}\right) \geq 0$. Suppose that $(\widetilde{u}-\widetilde{v})\left(x_{0}, t_{0}\right)<0$, on the contrary. Since $(\widetilde{u}-\widetilde{v})(x, t) \geq 0$ on both $\partial S \times\left[0, T^{\prime}\right]$ and $\bar{S} \times\{0\}$, we have $\left(x_{0}, t_{0}\right) \in S \times\left(0, T^{\prime}\right]$. Then we have

$$
\tilde{u}_{t}\left(x_{0}, t_{0}\right) \leq \widetilde{v}_{t}\left(x_{0}, t_{0}\right), \quad \Delta_{p, \omega} \widetilde{u}\left(x_{0}, t_{0}\right) \geq \Delta_{p, \omega} \widetilde{v}\left(x_{0}, t_{0}\right) .
$$

Since $\left|\xi^{p-2}(x, t)\right| \leq \max _{|r| \leq M}\left|r^{p-2}\right|=L$, we have

$$
\left[2 L-\left|\xi\left(x_{0}, t_{0}\right)\right|^{p-2}\right](\widetilde{u}-\widetilde{v})\left(x_{0}, t_{0}\right) \leq L(\widetilde{u}-\widetilde{v})\left(x_{0}, t_{0}\right)<0 .
$$

Combining (17) and (18), we obtain

$$
\begin{aligned}
& \tilde{u}_{t}\left(x_{0}, t_{0}\right)-\widetilde{v}_{t}\left(x_{0}, t_{0}\right)-e^{2 \lambda L(p-1)(p-2) t_{0}} \\
& \times\left[\Delta_{p, \omega} \widetilde{u}\left(x_{0}, t_{0}\right)-\Delta_{p, \omega} \widetilde{v}\left(x_{0}, t_{0}\right)\right]+\lambda(p-1) \\
& \quad \times\left[2 L-\left|\xi\left(x_{0}, t_{0}\right)\right|^{p-2}\right]\left[\widetilde{u}\left(x_{0}, t_{0}\right)-\widetilde{v}\left(x_{0}, t_{0}\right)\right]<0
\end{aligned}
$$

which contradicts (15). Therefore, $\widetilde{u}(x, t)-\widetilde{v}(x, t) \geq 0$ for all $(x, t) \in S \times\left(0, T^{\prime}\right]$ so that we get $u(x, t) \geq v(x, t)$ for all $(x, t) \in$ $\bar{S} \times[0, T)$, since $T^{\prime}<T$ is arbitrarily given. lary.

Now, we obtain a strict comparison principle as a corol-

Corollary 5 (strict comparison principle). In Theorem 4, if $u_{0}\left(x^{*}\right)>v_{0}\left(x^{*}\right)$ for some $x^{*} \in S$, then $u(x, t)>v(x, t)$ for all $(x, t) \in S \times(0, T)$.

Proof. First, note that $u \geq v$ on $\bar{S} \times[0, T)$ by Theorem 4 . Let $T^{\prime}>0$ be arbitrarily given with $T^{\prime}<T$ and let $\tau: \bar{S} \times[0$, $\left.T^{\prime}\right] \rightarrow \mathbb{R}$ be a function defined by

$$
\tau(x, t):=u(x, t)-v(x, t), \quad(x, t) \in \bar{S} \times\left[0, T^{\prime}\right] .
$$

Then $\tau(x, t) \geq 0$ for all $(x, t) \in \bar{S} \times\left[0, T^{\prime}\right]$. Since $\mid u\left(x^{*}\right.$, $t)\left.\right|^{p-2} u\left(x^{*}, t\right) \geq\left|v\left(x^{*}, t\right)\right|^{p-2} v\left(x^{*}, t\right)$ for all $0<t \leq T^{\prime}$, and $\tau\left(x^{*}, 0\right)>0$, we obtain from inequality (15) that

$$
\tau_{t}\left(x^{*}, t\right)-\left[\Delta_{p, \omega} u\left(x^{*}, t\right)-\Delta_{p, \omega} v\left(x^{*}, t\right)\right] \geq 0,
$$

for all $0<t \leq T^{\prime}$. Then by the mean value theorem, for each $y \in \bar{S}$ and $t$ with $0 \leq t \leq T^{\prime}$, it follows that

$$
\begin{aligned}
\left|u(y, t)-u\left(x^{*}, t\right)\right|^{p-2}\left[u(y, t)-u\left(x^{*}, t\right)\right] \\
\quad-\left|v(y, t)-v\left(x^{*}, t\right)\right|^{p-2}\left[v(y, t)-v\left(x^{*}, t\right)\right] \\
=(p-1)\left|\eta\left(x^{*}, y, t\right)\right|^{p-2}\left[\tau(y, t)-\tau\left(x^{*}, t\right)\right],
\end{aligned}
$$

and $\left|\eta\left(x^{*}, y, t\right)\right|<2 M$ where $M:=\max _{0<t \leq T^{\prime}}\left\{\left|u\left(x^{*}, t\right)\right|\right.$, $\left.\left|v\left(x^{*}, t\right)\right|\right\}$.

Then inequality (21) gives

$$
\begin{aligned}
\tau_{t}\left(x^{*}, t\right) \geq \sum_{y \in \bar{S}}(p-1)\left|\eta\left(x^{*}, y, t\right)\right|^{p-2} \\
\quad \times\left[\tau(y, t)-\tau\left(x^{*}, t\right)\right] \omega\left(x^{*}, y\right) \\
\geq-d(p-1)[2 M]^{p-2} \tau\left(x^{*}, t\right),
\end{aligned}
$$

where $d=\sum_{y \in \bar{S}} \omega\left(x^{*}, y\right)$. This implies

$$
\tau\left(x^{*}, t\right) \geq \tau\left(x^{*}, 0\right) e^{-d(p-1)(2 M)^{p-2} t}>0, \quad t \in\left[0, T^{\prime}\right] .
$$


Now, suppose there exists $\left(x_{0}, t_{0}\right) \in S \times\left(0, T^{\prime}\right]$ such that

$$
\tau\left(x_{0}, t_{0}\right)=\min _{x \in S, 0<t \leq T^{\prime}} \tau(x, t)=0 .
$$

Then

$$
\begin{gathered}
\tau_{t}\left(x_{0}, t_{0}\right) \leq 0, \\
\Delta_{p, \omega} u\left(x_{0}, t_{0}\right) \geq \Delta_{p, \omega} v\left(x_{0}, t_{0}\right) .
\end{gathered}
$$

Hence, inequality (21) gives

$$
0 \leq \tau_{t}\left(x_{0}, t_{0}\right)-\left[\Delta_{p, \omega} u\left(x_{0}, t_{0}\right)-\Delta_{p, \omega} v\left(x_{0}, t_{0}\right)\right] \leq 0 .
$$

Therefore,

$$
\Delta_{p, \omega} u\left(x_{0}, t_{0}\right)=\Delta_{p, \omega} v\left(x_{0}, t_{0}\right)
$$

that is,

$$
\begin{aligned}
& \sum_{y \in \bar{S}}\left\{\left|u\left(y, t_{0}\right)-u\left(x_{0}, t_{0}\right)\right|^{p-2}\left[u\left(y, t_{0}\right)-u\left(x_{0}, t_{0}\right)\right]\right. \\
& \left.\quad-\left|v\left(y, t_{0}\right)-v\left(x_{0}, t_{0}\right)\right|^{p-2}\left[v\left(y, t_{0}\right)-v\left(x_{0}, t_{0}\right)\right]\right\} \\
& \quad \times \omega\left(x_{0}, y\right)=0,
\end{aligned}
$$

which implies that $\tau\left(y, t_{0}\right)=0$ for all $y \in \bar{S}$ with $y \sim x_{0}$. Now, for any $x \in \bar{S}$, there exists a path

$$
x_{0} \sim x_{1} \sim \cdots \sim x_{n-1} \sim x_{n}=x,
$$

since $\bar{S}$ is connected. By applying the same argument as above inductively we see that $\tau\left(x, t_{0}\right)=0$ for every $x \in \bar{S}$. This gives a contradiction to (24).

For the case $1<p<2$, it is well known that (10) may not have unique solution, in general, and the comparison principle in usual form as in Theorem 4 may not hold. Instead, with a strict condition on the parabolic boundary we obtain a similar comparison principle as follows.

Theorem 6. Let $T>0$ ( $T$ may be $+\infty), \lambda>0$, and $p>1$. Suppose that real-valued functions $u(x, \cdot), v(x, \cdot) \in C[0, T)$ are differentiable in $(0, T)$ for each $x \in \bar{S}$ and satisfy

$$
\begin{gathered}
u_{t}(x, t)-\Delta_{p, \omega} u(x, t)-\lambda|u(x, t)|^{p-2} u(x, t) \\
\geq v_{t}(x, t)-\Delta_{p, \omega} v(x, t)-\lambda|v(x, t)|^{p-2} v(x, t), \\
(x, t) \in S \times(0, T), \\
u(x, t)>v(x, t), \quad(x, t) \in \partial S \times[0, T), \\
u(x, 0)>v(x, 0), \quad x \in \bar{S} .
\end{gathered}
$$

Then $u(x, t) \geq v(x, t)$ for all $(x, t) \in S \times(0, T)$.

Proof. Let $T^{\prime}>0$ and $\delta>0$ be arbitrarily given with $T^{\prime}<T$ and $0<\delta<\min _{(x, t) \in \Gamma}[u(x, t)-v(x, t)]$, respectively, where
$\Gamma:=\left\{(x, t) \in \bar{S} \times\left[0, T^{\prime}\right] \mid t=0\right.$ or $\left.x \in \partial S\right\}$ (called a parabolic boundary).

Now, let a function $\tau: \bar{S} \times\left(0, T^{\prime}\right] \rightarrow \mathbb{R}$ be a function defined by

$$
\tau(x, t):=[u(x, t)-v(x, t)]-\delta, \quad(x, t) \in \bar{S} \times\left(0, T^{\prime}\right] .
$$

Then $\tau(x, t)>0$ on $\Gamma$. Now, we suppose that $\min _{x \in S, 0<t \leq T^{\prime}}$ $\tau(x, t)<0$. Then there exists $\left(x_{0}, t_{0}\right) \in S \times\left(0, T^{\prime}\right]$ such that
(i) $\tau\left(x_{0}, t_{0}\right)=0$,
(ii) $\tau\left(y, t_{0}\right) \geq \tau\left(x_{0}, t_{0}\right)=0, y \in S$,
(iii) $\tau(x, t)>0,(x, t) \in S \times\left(0, t_{0}\right)$.

Then

$$
\tau_{t}\left(x_{0}, t_{0}\right) \leq 0, \quad \Delta_{p, \omega} u\left(x_{0}, t_{0}\right) \geq \Delta_{p, \omega} v\left(x_{0}, t_{0}\right),
$$

since $u\left(y, t_{0}\right)-u\left(x_{0}, t_{0}\right) \geq v\left(y, t_{0}\right)-v\left(x_{0}, t_{0}\right)$. Hence, inequality (31) gives

$$
\begin{aligned}
0 \geq \tau_{t} & \left(x_{0}, t_{0}\right) \\
\geq \lambda & {\left[\left|u\left(x_{0}, t_{0}\right)\right|^{p-2} u\left(x_{0}, t_{0}\right)-\left|v\left(x_{0}, t_{0}\right)\right|^{p-2}\right.} \\
& \left.\times v\left(x_{0}, t_{0}\right)\right] \\
=\lambda & {\left[\left|v\left(x_{0}, t_{0}\right)+\delta\right|^{p-2}\left(v\left(x_{0}, t_{0}\right)+\delta\right)\right.} \\
& \left.\quad-\left|v\left(x_{0}, t_{0}\right)\right|^{p-2} v\left(x_{0}, t_{0}\right)\right]>0,
\end{aligned}
$$

which leads to a contradiction. Hence, $\tau(x, t) \geq 0$ for all $(x, t) \in S \times\left(0, T^{\prime}\right]$ so that we have $u(x, t) \geq v(x, t)$ for all $(x, t) \in S \times(0, T)$, since $\delta$ and $T^{\prime}$ are arbitrary.

\section{Blow-Up and Global Existence}

In this section, we discuss the blow-up and global existence of the equation

$$
\begin{aligned}
& u_{t}(x, t)=\Delta_{p, \omega} u(x, t)+\lambda|u(x, t)|^{p-2} u(x, t), \\
& (x, t) \in S \times(0, \infty), \\
& u(x, t)=0, \quad(x, t) \in \partial S \times(0, \infty), \\
& u(x, 0)=u_{0} \geq 0, \quad x \in \bar{S},
\end{aligned}
$$

where $\lambda>0, p>1$, and the initial data $u_{0}$ is nontrivial on $S$. According to the comparison principle in the previous section, the nonhomogeneous term $\lambda|u(x, t)|^{p-2} u(x, t)$ can be written as a simpler form as $\lambda u^{p-1}(x, t)$, for the case where $p \geq 2$.

Definition 7 (blow-up). We say that a solution $u$ to an equation defined on a network $\bar{S}$ blows up in finite time $T$, if there exists $x \in S$ such that $|u(x, t)| \rightarrow+\infty$ as $t \nearrow T^{-}$. 
Theorem 8. For $p>2$ and $\lambda>0$, the solution to

$$
\begin{aligned}
& u_{t}(x, t)=\Delta_{p, \omega} u(x, t)+\lambda u^{p-1}(x, t), \quad(x, t) \in S \times(0, \infty), \\
& u(x, t)=0, \quad(x, t) \in \partial S \times(0, \infty), \\
& u(x, 0)=u_{0}(x) \geq 0 \text { (nontrivial) }
\end{aligned}
$$

blows up at some $T^{*}$, provided that

$$
-\frac{1}{2} \sum_{x, y \in \bar{S}}\left|u_{0}(x)-u_{0}(y)\right|^{p} \omega(x, y)+\lambda \sum_{x \in \bar{S}} u_{0}^{p}(x)>0 .
$$

Proof. First, we note that $u(x, t)>0$ on $S \times(0, \infty)$, by the strict comparison principle (Corollary 5). Now, we define a functional by

$$
\begin{aligned}
J(t):= & -\frac{1}{2 p} \sum_{x, y \in \bar{S}}|u(x, t)-u(y, t)|^{p} \omega(x, y) \\
& +\frac{\lambda}{p} \sum_{x \in \bar{S}}|u(x, t)|^{p}, \quad t \geq 0 .
\end{aligned}
$$

Then by (37),

$$
\begin{aligned}
J(0)= & -\frac{1}{2 p} \sum_{x, y \in \bar{S}}\left|u_{0}(x)-u_{0}(y)\right|^{p} \omega(x, y) \\
& +\frac{\lambda}{p} \sum_{x \in \bar{S}}\left|u_{0}(x)\right|^{p}>0 .
\end{aligned}
$$

Multiplying (36) by $u$ and summing up over $\bar{S}$, we obtain from Lemma 2

$$
\begin{aligned}
\frac{d}{d t} \sum_{x \in \bar{S}} u^{2}(x, t)= & -\sum_{x, y \in \bar{S}}|u(x, t)-u(y, t)|^{p} \omega(x, y) \\
& +2 \lambda \sum_{x \in \bar{S}} u^{p}(x, t) .
\end{aligned}
$$

Multiplying (36) by $u_{t}$ and summing up over $\bar{S}$, we obtain from Lemma 2

$$
\begin{gathered}
\sum_{x \in \bar{S}} u_{t}^{2}(x, t)=-\sum_{x \in \bar{S}} u_{t}(x, t)\left[-\Delta_{p, \omega} u(x, t)\right] \\
+\frac{d}{d t}\left[\frac{\lambda}{p} \sum_{x \in \bar{S}} u^{p}(x, t)\right], \\
\sum_{x \in \bar{S}} u_{t}(x, t)\left(-\Delta_{p, \omega} u(x, t)\right) \\
=\frac{1}{2} \sum_{x, y \in \bar{S}}|u(y, t)-u(x, t)|^{p-2} \\
\quad \times[u(y, t)-u(x, t)]\left[u_{t}(y)-u_{t}(x)\right]
\end{gathered}
$$

$$
\begin{aligned}
& \times \omega(x, y) \\
= & \frac{d}{d t}\left[\frac{1}{2 p} \sum_{x, y \in \bar{S}}|u(y, t)-u(x, t)|^{p} \omega(x, y)\right] .
\end{aligned}
$$

Then it follows that

$$
\begin{aligned}
\sum_{x \in \bar{S}} u_{t}^{2}(x, t)= & -\frac{d}{d t}\left[\frac{1}{2 p} \sum_{x, y \in \bar{S}}|u(y, t)-u(x, t)|^{p} \omega(x, y)\right] \\
& +\frac{d}{d t}\left[\frac{\lambda}{p} \sum_{x \in \bar{S}} u^{p}(x, t)\right] .
\end{aligned}
$$

Moreover, it follows from (42) that

$$
\begin{aligned}
J^{\prime}(t) & =\sum_{x \in \bar{S}} u_{t}^{2}(x, t), \\
J(t) & =\int_{0}^{t} J^{\prime}(s) d s+J(0)=\int_{0}^{t} \sum_{x \in \bar{S}} u_{t}^{2}(x, s) d s+J(0) .
\end{aligned}
$$

Now, we introduce a new function

$$
I(t)=\int_{0}^{t} \sum_{x \in \bar{S}} u^{2}(x, s) d s+M, \quad t \geq 0
$$

where $M>0$ is a constant to be determined later. Then by (40) we have

$$
\begin{gathered}
I^{\prime}(t)=\sum_{x \in \bar{S}} u^{2}(x, t), \\
I^{\prime \prime}(t)=\frac{d}{d t} \sum_{x \in \bar{S}} u^{2}(x, t) \\
=-\sum_{x, y \in \bar{S}}|u(x, t)-u(y, t)|^{p} \omega(x, y) \\
+2 \lambda \sum_{x \in \bar{S}} u^{p}(x, t) \\
=2 p\left[J(0)+\sum_{x \in \bar{S}} \int_{0}^{t} u_{t}^{2}(x, s) d s\right] .
\end{gathered}
$$

Moreover,

$$
\begin{aligned}
I^{\prime}(t)= & \sum_{x \in \bar{S}} u^{2}(x, t) \\
= & \sum_{x \in \bar{S}} \int_{0}^{t} 2 u(x, s) u_{t}(x, s) d s \\
& +\sum_{x \in \bar{S}} u_{0}^{2}(x) .
\end{aligned}
$$


Using the Schwarz inequality, we obtain

$$
\begin{aligned}
& \left\{I^{\prime}(t)\right\}^{2} \\
& \leq 4(1+\epsilon)\left[\sum_{x \in \bar{S}} \int_{0}^{t} u(x, s) u_{t}(x, s) d s\right]^{2} \\
& \quad+\left(1+\frac{1}{\epsilon}\right)\left[\sum_{x \in \bar{S}} u_{0}^{2}(x)\right]^{2} \\
& \leq 4(1+\epsilon)\left[\sum_{x \in \bar{S}}\left(\int_{0}^{t} u^{2}(x, s) d s\right)^{1 / 2}\left(\int_{0}^{t} u_{t}^{2}(x, s) d s\right)^{1 / 2}\right]^{2} \\
& \quad+\left(1+\frac{1}{\epsilon}\right)\left[\sum_{x \in \bar{S}} u_{0}^{2}(x)\right]^{2} \\
& \leq 4(1+\epsilon)\left(\sum_{x \in \bar{S}} \int_{0}^{t} u^{2}(x, s) d s\right)\left(\sum_{x \in \bar{S}} \int_{0}^{t} u_{t}^{2}(x, s) d s\right) \\
& +\left(1+\frac{1}{\epsilon}\right)\left[\sum_{x \in \bar{S}} u_{0}^{2}(x)\right]^{2},
\end{aligned}
$$

where $\epsilon>0$ is arbitrary. Combining the above estimates (46), (47), and (48), we obtain that, for $\alpha=\epsilon=\sqrt{p / 2}-1>0$,

$$
\begin{array}{rl}
I^{\prime \prime}(t) & I(t)-(1+\alpha) I^{\prime}(t)^{2} \\
\geq & 2 p\left[J(0)+\sum_{x \in \bar{S}} \int_{0}^{t} u_{t}^{2}(x, s) d s\right] \\
& \times\left[\sum_{x \in \bar{S}} \int_{0}^{t} u^{2}(x, s) d s+M\right] \\
& -4(1+\alpha)(1+\epsilon)\left[\sum_{x \in \bar{S}} \int_{0}^{t} u^{2}(x, s) d s\right] \\
& \times\left[\sum_{x \in \bar{S}} \int_{0}^{t} u_{t}^{2}(x, s) d s\right] \\
& \left.-(1+\alpha)\left(1+\frac{1}{\epsilon}\right)\left[\sum_{x \in \bar{S}} u_{0}^{2}(x)\right]^{2}\right]^{2} \\
\geq 2 & 2 p M \cdot J(0)-(1+\alpha)\left(1+\frac{1}{\epsilon}\right)\left[\sum_{x \in \bar{S}} u_{0}^{2}(x)\right]^{2} .
\end{array}
$$

Since $J(0)>0$ by assumption, we can choose $M>0$ to be large enough so that

$$
I^{\prime \prime}(t) I(t)-(1+\alpha) I^{\prime}(t)^{2}>0 .
$$

Thus inequality (50) implies that, for $t \geq 0$,

$$
\begin{aligned}
& \frac{d}{d t}\left[\frac{I^{\prime}(t)}{I^{\alpha+1}(t)}\right]>0 \\
& \text { i.e., } I^{\prime}(t) \geq\left[\frac{I^{\prime}(0)}{I^{\alpha+1}(0)}\right] I^{\alpha+1}(t) .
\end{aligned}
$$

Therefore, it follows that $I(t)$ cannot remain finite for all $t>0$. In other words, the solutions $u(x, t)$ blow up at some time $T^{*}$.

Remark 9. (i) Condition (37) implies that

$$
\lambda>\frac{(1 / 2) \sum_{x, y \in \bar{S}}\left|u_{0}(x)-u_{0}(y)\right|^{p} \omega(x, y)}{\sum_{x \in \bar{S}} u_{0}^{p}(x)} \geq \lambda_{0},
$$

where $\lambda_{0}$ is the first eigenvalue of $\Delta_{p, \omega}$.

(ii) The initial data $u_{0}$ with $J(0)>0$ always exists. In fact, consider an eigenvalue $\lambda_{0}>0$ and eigenfunction $\phi_{0}(x)>0$ in Lemma 3. Taking $u_{0}(x)=\phi_{0}(x)$, then we have

$$
\begin{aligned}
J(0) & =-\frac{1}{p} \sum_{x \in \bar{S}} \phi_{0}(x)\left[-\Delta_{p, \omega} \phi_{0}(x)\right]+\frac{\lambda}{p} \sum_{x \in \bar{S}} \phi_{0}^{p}(x) \\
& =\frac{\lambda-\lambda_{0}}{p} \sum_{x \in \bar{S}} \phi_{0}^{p}(x)>0,
\end{aligned}
$$

where $\lambda>\lambda_{0}$.

(iii) When the solution to (37) is global, then we must have

$$
\begin{aligned}
J(t)= & -\frac{1}{2 p} \sum_{x, y \in \bar{S}}|u(x, t)-u(y, t)|^{p} \omega(x, y) \\
& +\frac{\lambda}{p} \sum_{x \in \bar{S}}|u(x, t)|^{p}<0
\end{aligned}
$$

for all $t>0$.

(iv) The blow-up time in the above can be estimated roughly. Taking

$$
M:=\frac{(p /(p-2))(1+\sqrt{p / 2})\left[\sum_{x \in \bar{S}} u_{0}^{2}(x)\right]^{2}}{-\sum_{x, y \in \bar{S}}\left|u_{0}(x)-u_{0}(y)\right|^{p} \omega(x, y)+2 \lambda \sum_{x \in \bar{S}}\left|u_{0}(x)\right|^{p}}
$$

we see that

$$
\begin{aligned}
& I^{\prime}(t) \geq\left[\frac{\sum_{x \in \bar{S}} u_{0}^{2}(x)}{M^{\alpha+1}}\right] I^{\alpha+1}(t), \quad t>0, \\
& I(0)=M,
\end{aligned}
$$

which implies

$$
I(t) \geq\left[\frac{1}{M^{\alpha}}-\frac{\alpha \sum_{x \in \bar{S}} u_{0}^{2}(x)}{M^{\alpha+1}} t\right]^{-(1 / \alpha)},
$$

where $\alpha=\sqrt{p / 2}-1>0$. Then the blow-up time $T^{*}$ satisfies

$$
0<T^{*} \leq \frac{M}{\alpha \sum_{x \in \bar{S}} u_{0}^{2}(x)} .
$$

Now, we state the main theorem as follows. 
Theorem 10. The solution to (36) with $p>2$ and $\lambda>\lambda_{0}$ blows up in finite time, for every nonnegative and nontrivial initial data $u_{0}$.

Proof. First, we note that (36) has a unique solution such that

$$
u(x, t)>0, \quad x \in S, t>0 .
$$

Take $t_{0}>0, k>0$ arbitrarily and consider an equation

$$
\begin{aligned}
v_{t}(x, t) & =\Delta_{p, \omega} v(x, t)+\lambda v^{p-1}(x, t), \quad x \in S, t>t_{0}, \\
v(x, t) & =0, \quad x \in \partial S, t \geq t_{0} \\
v\left(x, t_{0}\right) & =k \phi_{0}(x), \quad x \in S,
\end{aligned}
$$

where $\phi_{0}$ is the eigenfunction corresponding to the first eigenvalue $\lambda_{0}>0$. Then the initial data $v\left(x, t_{0}\right)$ satisfies

$$
\begin{aligned}
& -\frac{1}{2} \sum_{x, y \in \bar{S}}\left|v\left(x, t_{0}\right)-v\left(y, t_{0}\right)\right|^{p} \omega(x, y)+\lambda \sum_{x \in \bar{S}} v^{p}\left(x, t_{0}\right) \\
& \quad=k^{p}\left(\lambda-\lambda_{0}\right) \sum_{x \in \bar{S}} \phi_{0}^{p}(x)>0 .
\end{aligned}
$$

Then, by Theorem 8 , the solution $v(x, t)$ is positive and blows up in a finite time. Since

$$
\begin{aligned}
& u(x, t)=0=v(x, t), \quad x \in S, t \geq t_{0}, \\
& u\left(x, t_{0}\right)>v\left(x, t_{0}\right)=k \phi_{0}(x), \quad x \in S
\end{aligned}
$$

for a small $k>0$, the comparison principle enables us to see

$$
0<v(x, t) \leq u(x, t) \quad x \in S, t \geq t_{0},
$$

which completes the proof.

Remark 11. According to Remark 9 (iv) to Theorem 8, the blow-up time for the solution to (36) is estimated by

$$
0<T^{*} \leq \frac{p(1+\sqrt{p / 2})^{2}}{k^{p-2}(p-2)^{2}\left(\lambda-\lambda_{0}\right)}\left[\sum_{x \in \bar{S}} \phi_{0}^{2}(x)\right]^{2} .
$$

We now derive the lower bound for the maximum function of blow-up solutions.

Theorem 12. Let $u$ be the solutions to (36) blowing up at finite time $T$. Then it follows that

$$
\max _{x \in \bar{S}} u(x, t) \geq\left[(p-2)\left(\lambda-\lambda_{0}\right)(T-t)\right]^{-(1 /(p-2))}
$$

for all $t>0$.

Proof. For each $t>0$, let $x_{t} \in S$ be the node such that

$$
u\left(x_{t}, t\right)=\max _{x \in \bar{S}} u(x, t) .
$$

In fact, we note that $u\left(x_{t}, t\right)$ is continuous on $[0, \infty)$ and differentiable for almost all $t>0$. Then (36) can be written as

$$
\begin{aligned}
u_{t}\left(x_{s}, s\right)= & \Delta_{p, \omega} u\left(x_{s}, s\right)+\lambda u^{p-1}\left(x_{s}, s\right) \\
= & \sum_{y \in \bar{S}}\left|u(y, s)-u\left(x_{s}, s\right)\right|^{p-2} \\
& \times\left[u(y, s)-u\left(x_{s}, s\right)\right] \omega\left(x_{s}, y\right) \\
& +\lambda u^{p-1}\left(x_{s}, s\right)
\end{aligned}
$$

for almost all $s>0$.

Now define a function $v: \bar{S} \times[0, \infty) \rightarrow[0, \infty)$ by

$$
v(x, t):= \begin{cases}u\left(x_{t}, t\right), & x \in S, t \geq 0 \\ 0, & x \in \partial S, t \geq 0 .\end{cases}
$$

Then $v(x, t)=v(y, t), t>0$, if $x, y \in S$. Multiplying (67) by $u\left(x_{s}, s\right)$ and summing over $\bar{S}$, we obtain, for almost all $t>0$,

$$
\begin{aligned}
\sum_{x \in \bar{S}} v_{t}(x, t) v(x, t) & \\
& =\sum_{x \in \bar{S}} v(x, t) \Delta_{p, \omega} v(x, t)+\lambda \sum_{x \in \bar{S}} v^{p}(x, t) \\
& \leq\left(\lambda-\lambda_{0}\right) \sum_{x \in \bar{S}} v^{p}(x, t)
\end{aligned}
$$

or, equivalently,

$$
v_{t}(x, t) \leq\left(\lambda-\lambda_{0}\right) v^{p-1}(x, t), \quad x \in S .
$$

Integrating over $[t, T]$, we have

$$
v(x, t) \geq\left[(p-2)\left(\lambda-\lambda_{0}\right)(T-t)\right]^{-(1 /(p-2))},
$$

which is desired.

Now, we state the global existence of the solutions.

Theorem 13. For $p>2$ and $\lambda \leq \lambda_{0}$, the solution to (36) is global for every nonnegative initial data $u_{0}$.

Proof. Consider an eigenvalue $\lambda_{0}>0$ and eigenfunction $\phi_{0}(x)>0$ in Lemma 3 and $v(x, t):=K \phi_{0}(x), x \in \bar{S}, t \geq 0$. Then by taking $K$ large enough to be $K \phi_{0}(x) \geq u_{0}(x), x \in S$, we have

$$
\begin{gathered}
v(x, 0) \geq u(x, 0), \quad x \in \bar{S}, \\
v_{t}(x, t)-\Delta_{p, \omega} v(x, t)-\lambda v^{p-1}(x, t) \\
=\left(\lambda_{0}-\lambda\right) K^{p-1} \phi_{0}(x) \geq 0
\end{gathered}
$$

for all $t>0$ and $x \in S$. Then by the comparison principle, we see that $0 \leq u(x, t) \leq K \phi_{0}(x), x \in S, t \geq 0$, which is desired. 
We have so far discussed the blow-up or the global existence (35) for the case $p \geq 2$. Now, we discuss the case $1<p \leq 2$. Here, we note that when $1<p<2$, the solution to the equation may not have unique positive solution.

Theorem 14. For $1<p \leq 2$, the nonnegative solution to

$$
\begin{aligned}
& u_{t}(x, t)=\Delta_{p, \omega} u(x, t)+\lambda u^{p-1}(x, t), \quad(x, t) \in S \times(0, \infty), \\
& u(x, t)=0, \quad(x, t) \in \partial S \times(0, \infty), \\
& u(x, 0)=u_{0}(x) \geq 0
\end{aligned}
$$

is global for every $\lambda>0$. In particular, when $1<p<2$ and $\lambda<\lambda_{0}$, then there exists $T$ (extinction time) such that $u(x, t) \equiv$ 0 for $t \geq T$.

Proof. First, consider ODE

$$
\begin{gathered}
\frac{d}{d t} z(t)=\lambda z^{p-1}, \quad t>0, \\
z(0)=\bar{u}_{0}+1,
\end{gathered}
$$

where $\bar{u}_{0}=\max _{x \in \bar{S}} u_{0}(x)>0$. Then

$$
z(t)= \begin{cases}{\left[(2-p) t+\lambda z^{2-p}(0)\right]^{1 /(2-p)},} & 1<p<2, \\ \lambda z(0) e^{t}, & p=2 .\end{cases}
$$

Then by the comparison principle (Theorem 6 ), we see that

$$
0 \leq u(x, t) \leq z(t), \quad x \in S, t \geq 0,
$$

which implies that $u(x, t)$ must be global.

Now, assume $1<p<2$ and $\lambda<\lambda_{0}$. When $u$ is a trivial solution, then we are done. So now we assume that $u$ is nontrivial. Multiplying (73) by $u$, as done in (40), we have

$$
\begin{aligned}
\frac{1}{2} \frac{d}{d t} \sum_{x \in \bar{S}} u^{2}(x, t) \\
=-\frac{1}{2} \sum_{x, y \in \bar{S}}|u(x, t)-u(y, t)|^{p} \omega(x, y) \\
\quad+\lambda \sum_{x \in \bar{S}} u^{p}(x, t) \\
\leq-\left(\lambda_{0}-\lambda\right) \sum_{x \in \bar{S}} u^{p}(x, t) \\
\leq-\left(\lambda_{0}-\lambda\right)\left[\sum_{x \in \bar{S}} u^{2}(x, t)\right]^{p / 2},
\end{aligned}
$$

where the last inequality follows from the elementary inequality

$$
\left[\sum_{j=1}^{n} t_{j}^{p}\right]^{1 / p} \geq\left[\sum_{j=1}^{n} t_{j}^{2}\right]^{1 / 2}, \quad 1<p<2
$$

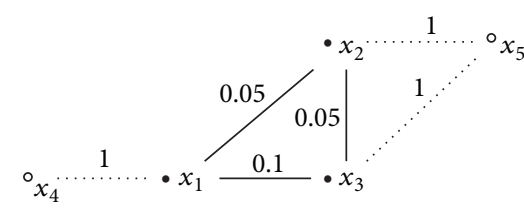

Figure 1: Graph $\bar{S}$.

for $t_{1}, t_{2}, \ldots, t_{n} \geq 0$. Now suppose $y(t):=\sum_{x \in S} u^{2}(x, t)>0$ for $t \geq 0$, on the contrary. Since $u(x, t)=0, x \in \partial S, t \geq 0$, we obtain

$$
\begin{aligned}
& y^{\prime}(t) \leq-2\left(\lambda_{0}-\lambda\right) y^{p / 2}, \quad t>0, \\
& y(0)=\sum_{x \in S} u_{0}^{2}(x)>0 .
\end{aligned}
$$

Integrating (79) over $[0, t]$, we obtain

$$
0<y^{(1-(p / 2))}(t) \leq y^{(1-(p / 2))}(0)-(2-p)\left(\lambda_{0}-\lambda\right) t,
$$

which is absurd for large $t>0$. Hence, $y(T)=0$ for some $T>0$ and, therefore, we can conclude that $y(t)=0, t>T$, for some $T>0$, since $y^{\prime}(t) \leq 0$ in inequality (79).

Remark 15. (i) When $p=2$ in the above, we do not need the assumption that $u$ is nonnegative, which follows automatically from the comparison principle (Theorem 4).

(ii) In fact, inequality (80) gives us the extinction time $T$, estimated by

$$
0<T \leq \frac{\left[\sum_{x \in S} u_{0}^{2}(x)\right]^{(1-(p / 2))}}{(2-p)\left(\lambda_{0}-\lambda\right)} .
$$

\section{Examples and Numerical Illustrations}

In this section, we show numerical illustrations to exploit our results in the previous section.

Now, consider a graph $S=\left\{x_{1}, x_{2}, x_{3}\right\}$ with the boundary $\partial S=\left\{x_{4}, x_{5}\right\}$ and the weight

$$
\begin{aligned}
& \omega\left(x_{1}, x_{2}\right)=\omega\left(x_{2}, x_{3}\right)=0.05, \\
& \omega\left(x_{1}, x_{3}\right)=0.1 \\
& \omega\left(x_{1}, x_{4}\right)=\omega\left(x_{2}, x_{5}\right)=\omega\left(x_{3}, x_{5}\right)=1
\end{aligned}
$$

as in Figure 1.

Example 1. For (36) on (Figure 1) graph $\bar{S}$ with $p=3, \lambda=1.1$, consider initial data given by $u_{0}\left(x_{1}\right)=20, u_{0}\left(x_{2}\right)=30$, and $u_{0}\left(x_{3}\right)=10$. Then by easy calculation, we get the first eigenvalue $\lambda_{0}=1<\lambda=1.1$ and the corresponding eigenfunction $\phi_{0}\left(x_{1}\right)=\phi_{0}\left(x_{2}\right)=\phi_{0}\left(x_{3}\right) \fallingdotseq 0.6934$. Figure 2 shows that the solution to (36) blows up and the computed blow-up time $T$ is estimated as $T \fallingdotseq 0.4433079$.

On the other hand, consider the same equation (36) with $p=3, \lambda=0.9$ and the same initial data. Then $\lambda=0.9<\lambda_{0}=$ 1 and Figure 3 shows that the solution to (36) is global. 


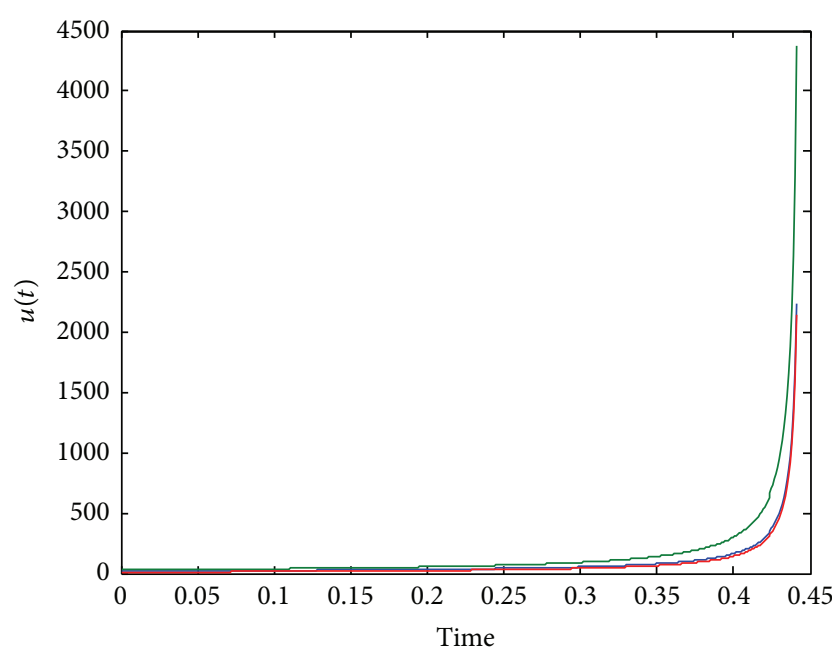

FIgURE 2: Behavior of each node for $p=3$ and $\lambda=1.1$.

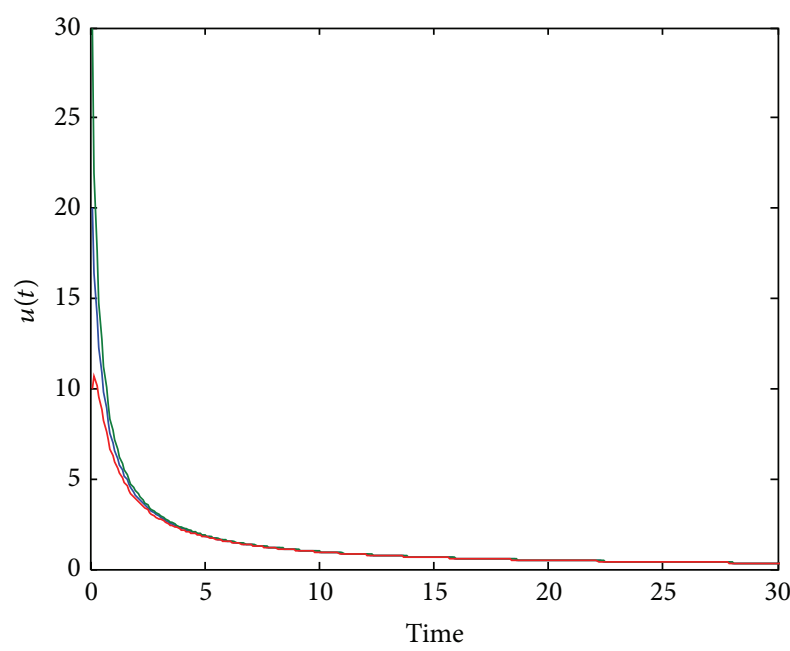

Figure 3: Behavior of each node for $p=3$ and $\lambda=0.9$.

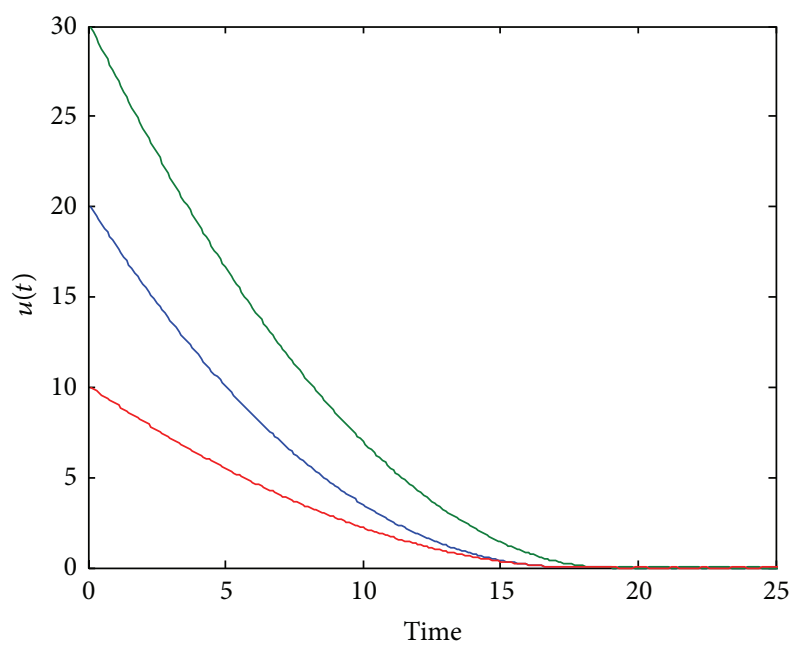

FIGURE 4: Behavior of each node for $p=1.5$.
Example 2. For (36) on (Figure 1) graph $\bar{S}$ with $p=1.5$, $\lambda=0.5$, and the same initial data $u_{0}$ given by $u_{0}\left(x_{1}\right)=20$, $u_{0}\left(x_{2}\right)=30$, and $u_{0}\left(x_{3}\right)=10$, then Figure 4 shows that the solution to (36) is global and extinctive.

\section{Conflict of Interests}

The author declares that there is no conflict of interests regarding the publication of this paper.

\section{Acknowledgments}

This work was supported by the National Research Foundation of Korea (NRF) grant funded by the Korean government (MOE) (no. 2012R1A1A2004689) and Sogang University Research Grant of 2014 (no. 201410044).

\section{References}

[1] A. Fujii and M. Ohta, "Asymptotic behavior of blowup solutions of a parabolic equation with the $p$-Laplacian," Publications of the Research Institute for Mathematical Sciences, vol. 32, no. 3, pp. 503-515, 1996.

[2] B. Hu, Blow-up Theories for Semilinear Parabolic Equations, vol. 2018 of Lecture Notes in Mathematics, Springer, Berlin, Germany, 2011.

[3] W. Liu, "A note on blow-up for fast diffusive $p$-Laplacian with sources," Mathematical Methods in the Applied Sciences, vol. 32, no. 2, pp. 241-245, 2009.

[4] Y. Li and C. Xie, "Blow-up for $p$-Laplacian parabolic equations," Electronic Journal of Differential Equations, no. 20, pp. 1-12, 2003.

[5] M. Tsutsumi, "Existence and nonexistence of global solutions for nonlinear parabolic equations," Research Institute for Mathematical Sciences. Publications, vol. 8, no. 2, pp. 211-229, 1972.

[6] J. Yin and C. Jin, "Critical extinction and blow-up exponents for fast diffusive $p$-Laplacian with sources," Mathematical Methods in the Applied Sciences, vol. 30, no. 10, pp. 1147-1167, 2007.

[7] J. Zhao and Z. Liang, "Blow-up rate of solutions for $p$-Laplacian equation," Journal of Partial Differential Equations, vol. 21, no. 2, pp. 134-140, 2008.

[8] S.-Y. Chung and C. A. Berenstein, " $\omega$-harmonic functions and inverse conductivity problems on networks," SIAM Journal on Applied Mathematics, vol. 65, no. 4, pp. 1200-1226, 2005.

[9] Q. Xin, L. Xu, and C. Mu, "Blow-up for the $\omega$-heat equation with Dirichlet boundary conditions and a reaction term on graphs," Applicable Analysis, vol. 93, no. 8, pp. 1691-1701, 2014.

[10] Y.-S. Chung, Y.-S. Lee, and S.-Y. Chung, "Extinction and positivity of the solutions of the heat equations with absorption on networks," Journal of Mathematical Analysis and Applications, vol. 380, no. 2, pp. 642-652, 2011.

[11] Y.-S. Lee and S.-Y. Chung, "Extinction and positivity of solutions of the $p$-Laplacian evolution equation on networks," Journal of Mathematical Analysis and Applications, vol. 386, no. 2, pp. 581-592, 2012.

[12] F. R. Chung, Spectral Graph Theory, CBMS Regional Conference Series in Mathematics, American Mathematical Society, 1997.

[13] D. M. Cvetković, M. Doob, and H. Sachs, Spectra of Graphs: Theory and Applications, Academic Press, New York, NY, USA, 1980. 
[14] J.-H. Park and S.-Y. Chung, "Positive solutions for discrete boundary value problems involving the $p$-laplacian with potential terms," Computers \& Mathematics with Applications, vol. 61, no. 1, pp. 17-29, 2011.

[15] J.-H. Park and S.-Y. Chung, "The Dirichlet boundary value problems for $p$-Schrödinger operators on finite networks," Journal of Difference Equations and Applications, vol. 17, no. 5, pp. 795-811, 2011. 


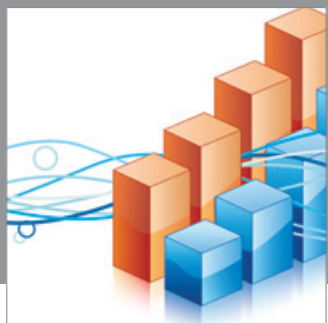

Advances in

Operations Research

mansans

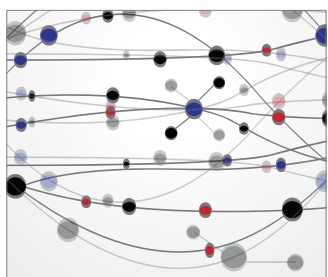

The Scientific World Journal
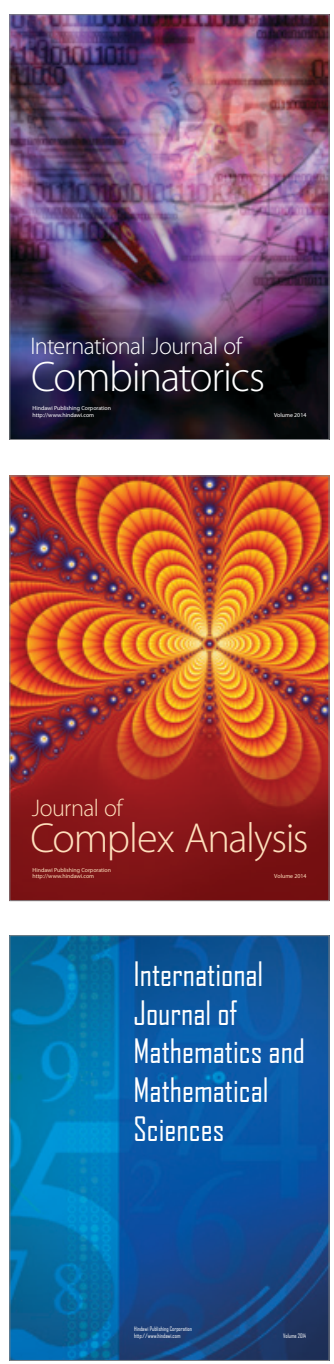
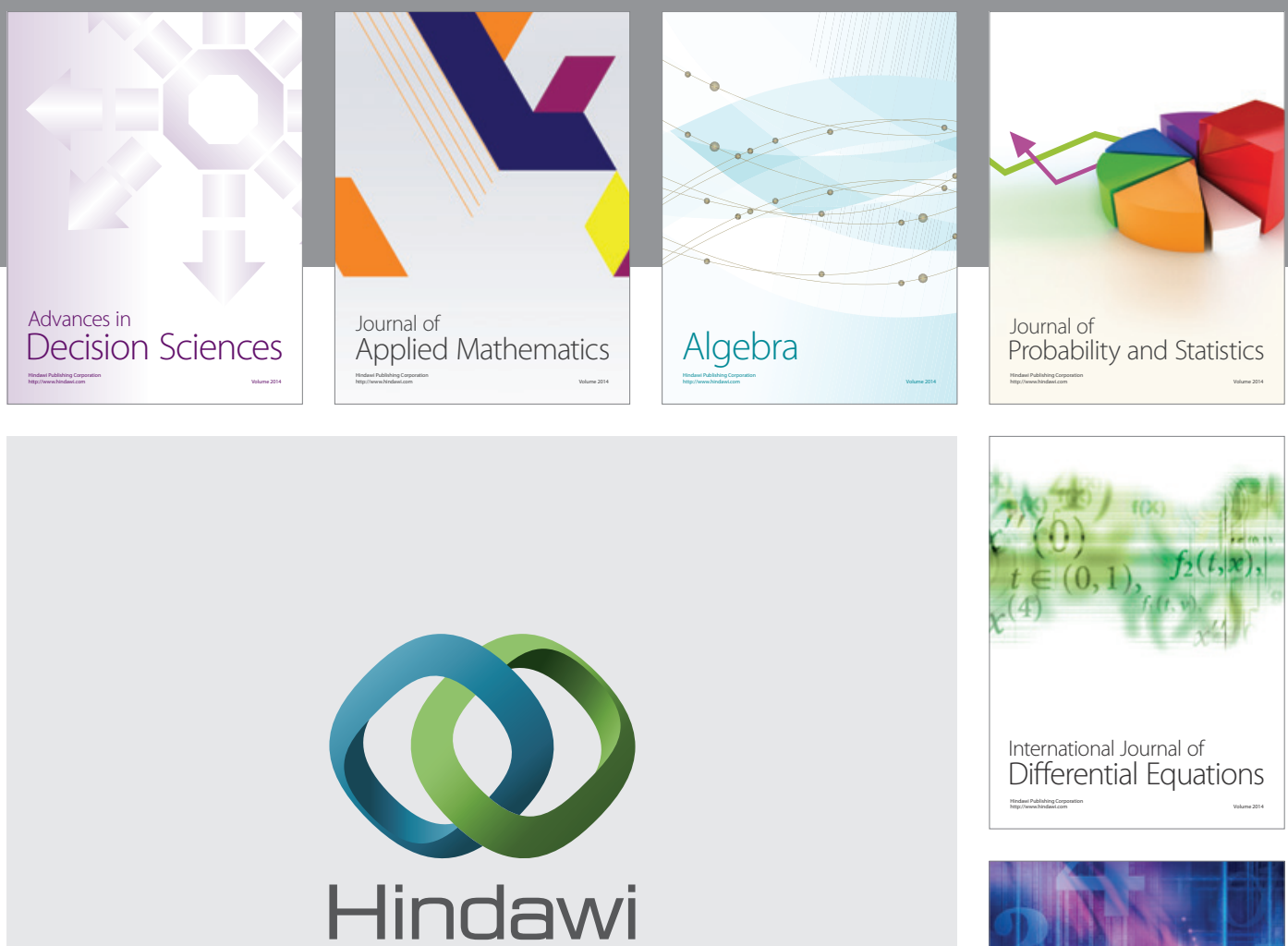

Submit your manuscripts at http://www.hindawi.com
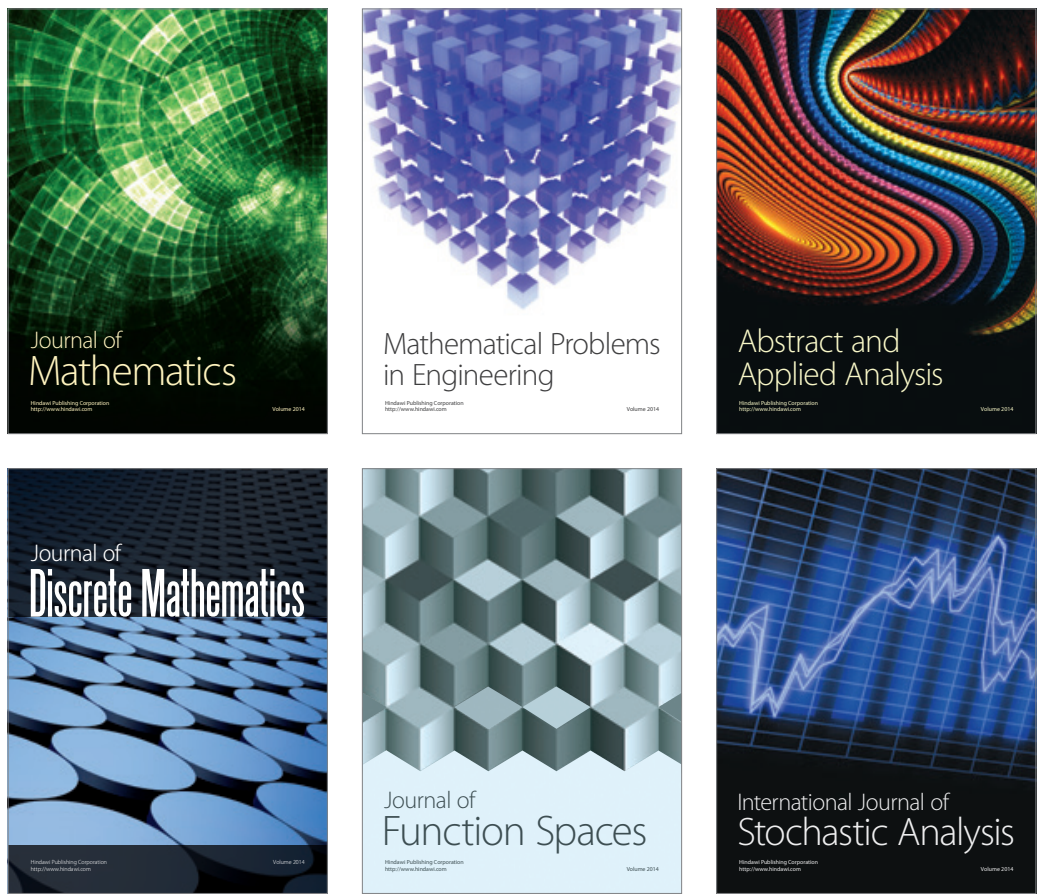

Journal of

Function Spaces

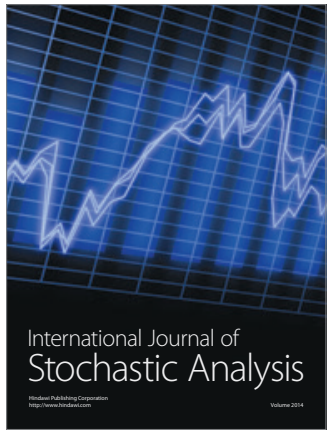

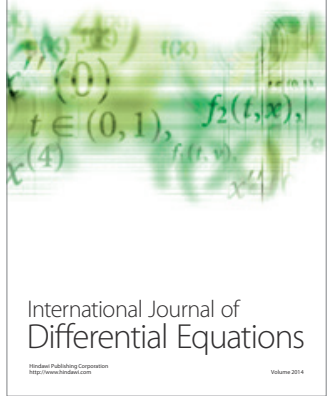
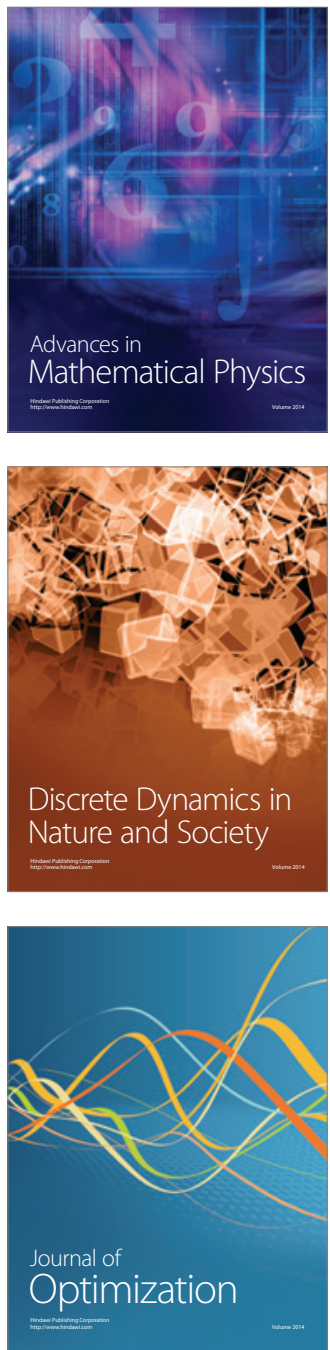